\title{
Glucose tolerance and plasma insulin response to intravenous glucose infusion and test meal in rats with microencapsulated islet allografts
}

\author{
W.M. Fritschy ${ }^{1}$, J.H.Strubbe ${ }^{2}$, G.H.J. Wolters ${ }^{1}$ and R.van Schilfgaarde ${ }^{1}$ \\ ${ }^{1}$ Department of Surgery, University of Groningen, Groningen, and ${ }^{2}$ Department of Animal Physiology, University of Groningen, Haren, \\ The Netherlands
}

\begin{abstract}
Summary. Albino Oxford rats made diabetic with $75 \mathrm{mg} / \mathrm{kg}$ streptozotocin were intraperitoneally transplanted with 2500-2900 alginate-polylysine microencapsulated Lewis islets $(n=9$, total islet tissue volume 8.0-11.0 $\mu \mathrm{l})$, or a similar volume of non-encapsulated Lewis islets $(n=5)$. All rats with microencapsulated islets became normoglycaemic, and remained normoglycaemic for 5-16 weeks. In rats with non-encapsulated islet grafts, only a temporary decrease in blood glucose was observed, and all were again severely hyperglycaemic at 1 week after implantation. At 5-6 weeks after transplantation, glucose tolerance in rats with microencapsulated islets was tested by intravenous glucose infusion $(10 \mathrm{mg} / \mathrm{min}$ over $20 \mathrm{~min})$ and test meal administration $(n=4)$. During glucose infusion, maximum glucose levels were $13.0 \pm 0.4 \mathrm{mmol} / 1$ in rats with microcapsules and $8.9 \pm 0.4 \mathrm{mmol} / \mathrm{l}$ in healthy control rats $(p<0.01)$. Concomi-
\end{abstract}

tant maximum plasma insulin levels were $215 \pm 17 \mathrm{pmol} / \mathrm{l}$ in rats with microcapsules and $715 \pm 85 \mathrm{pmol} / \mathrm{l}$ in controls $(p<0.001)$. After the test meal, maximum blood glucose was $10.6 \pm 0.9 \mathrm{mmol} / \mathrm{l}$ in rats with microcapsules and $6.2 \pm$ $0.1 \mathrm{mmol} / 1$ in controls $(p<0.001)$, with concomitant maximum plasma insulin levels of $247 \pm 11 \mathrm{pmol} / 1$ and $586 \pm$ $59 \mathrm{pmol} / \mathrm{l}$, respectively $(p<0.001)$. In conclusion, although the glucose tolerance is impaired and plasma insulin responses to intravenous glucose-load and test-meal are reduced, the alginate-polylysine membrane does provide adequate immunoisolation for the prolongation of allograft survival, resulting in prolonged normoglycaemia in streptozotocin diabetic rats.

Key words: Glucose tolerance, streptozotocin, diabetic rats, microencapsulation, islet transplantation.
The advantage of pancreatic islet transplantation over exogenous insulin administration in the treatment of diabetes mellitus is, conceptually, the minute-to-minute control of glucose metabolism by an endogenous insulin source. It is thought that close regulation of glucose and insulin levels may help to prevent long-term diabetic complications. However, after 20 years of intensive research, islet transplantation is still not readily available for Type 1 (insulin-dependent) diabetic patients. The main causes are 1) that islet isolation techniques are inappropriate for obtaining large amounts of islet tissue, and 2) a lack of appropriate means to prevent graft rejection after transplantation [1-3].

A possible method to circumvent rejection may be immuno-isolation of islets in a biocompatible membrane. By this technique, the encapsulated tissue is separated from the diabetic host by an artificial membrane which has to be permeable to glucose and insulin but not to immunologically active cells and cytotoxic antibodies [4, 5]. In 1980, Lim and Sun described the first successful transplantation of rat islets microencapsulated in a cross- linked polyamino-acid alginate membrane [6]. Several other publications followed, in which it was reported that prolonged normoglycaemia could be achieved with alginate-polylysine microencapsulated islets after allo- [7, 8] or xeno-transplantation [9] in streptozotocin diabetic rats and mice, respectively. Recently, it has been demonstrated that implantation of microencapsulated islet grafts could also reverse hyperglycaemia in auto-immune diabetic rats [10] and mice [11], although the duration of normoglycaemia in these animal models was generally shorter than could be achieved in chemically induced diabetic animals. However, the normalization of the plasma insulin levels, and in particular the capability of an intraperitoneal microencapsulated islet graft to increase plasma insulin in response to blood glucose elevation has never been detailed. Therefore, in the present study we investigated blood glucose levels and plasma insulin responses during intravenous glucose infusion and after test-meal administration in streptozotocin diabetic rats successfully transplanted with a microencapsulated islet allograft. 


\section{Materials and methods}

\section{Animals and islet isolation}

Male rats of the highly inbred Albino Oxford strain $(\mathrm{AO} / \mathrm{G}$, weight $280-320 \mathrm{~g}$ ) were used as islet graft recipients. Diabetes was induced by intravenous injection of streptozotocin $(75 \mathrm{mg} / \mathrm{kg}$, Zanosar, a gift from Upjohn Company, Kalamazoo, Mich., USA). Rats were classified as diabetic when they developed severe weight loss, polyuria, and non-fasting blood glucose levels exceeding $20 \mathrm{mmol} / 1$ over a period of at least 2 weeks.

Donor islets were isolated from highly inbred Lewis rats (Harlan CPB, Zeist, The Netherlands, weight 300-350 g). Under ether anaesthesia, the abdomen was opened by a midline incision and the common bile duct was identified and cannulated. The pancreas was distended with $10 \mathrm{ml}$ Krebs' Ringer solution containing $10 \%$ bovine serum albumin, excised, and cut into small pieces. A two-stage collagenase incubation at $37^{\circ} \mathrm{C}$ was performed with 1.2 and $0.7 \mathrm{mg} / \mathrm{ml}$ collagenase respectivly (Sigma type XI, $1800 \mathrm{U} / \mathrm{mg}$; Sigma, Chemical Company, St. Louis, Mo., USA) [12]. The islets were separated from the exocrine elements by means of a 5-layer discontinuous dextran gradient. Subsequently, $900-1200$ islets per donor pancreas were hand-picked, in order to obtain a pure islet preparation. A $10 \%$ sample was taken for measuring the diameters of the islets and to calculate the total islet graft volume. All procedures were performed under sterile conditions and with sterilized media.

\section{Microencapsulation}

Immediately after isolation, the islets were encapsulated in an alginate-polylysine membrane according to a modified method of $\mathrm{Lim}$ [13] as previously published in detail [14]. Briefly, the islets were suspended in a $2.0 \%$ (weight/volume) sodium alginate solution in $\mathrm{Ca}^{2+}$. free Krebs' Ringer Hepes (Algin, lot 23814, Kelco, San Diego, Calif., USA, dry-sterilized with ethylene oxide). Islet-containing alginate droplets were produced with an air-jet droplet generator, and hardened in a $100 \mathrm{mmol} / \mathrm{l} \mathrm{CaCl}$-solution. After several washes with Krebs' Ringer Hepes buffer ( $\mathrm{KRH}, \mathrm{O}_{2}$-gassed, $\mathrm{pH}$ 7.4), a polylysine membrane was formed by suspending the gelled alginate beads in a $0.05 \%$ polylysine solution for $6-9 \mathrm{~min}$ (poly-l-lysine- $\mathrm{HCl}, \mathrm{M}_{\mathrm{r}} 22,000$, Sigma). Subsequently, to apply the outer alginate layer, the beads were washed with $\mathrm{Ca}^{2+}$-free $\mathrm{KRH}$ and suspended in a $0.02 \%$ sodium alginate solution for 3-5 min. In order to liquify the inner core of the alginate polylysine beads, the beads were suspended in $1 \mathrm{mmol} / 1$ EGTA in $\mathrm{Ca}^{2+}$-free $\mathrm{KRH}$ for $10 \mathrm{~min}$. After three final washes in $\mathrm{Ca}^{2+}$-free $\mathrm{KRH}$, the microencapsulated islets were transferred to tissue culture Petri dishes containing RPMI culture medium, penicillin $\mathrm{G}(100 \mathrm{U} / \mathrm{ml})$, streptomycin $(100 \mu \mathrm{g} / \mathrm{ml})$, and $10 \mathrm{mmol} / 1$ glucose. Transplantation was performed immediately after encapsulation.

\section{Transplantation protocol}

A total of nine diabetic rats were transplanted with a microencapsulated islet graft, and five diabetic rats received a non-encapsulated islet graft. Prior to implantation, the microencapsulated islet grafts were carefully inspected under a stereomicroscope with side-illumination to confirm complete encapsulation of the islets. Each transplantation was performed with $2500-2900$ islets with a calculated total islet tissue volume of $8.00-11.0 \mu \mathrm{l}$. Uniform smooth spherical capsules were prepared, with diameters of $800-900 \mu \mathrm{m}$. Each capsule contained 1-3 islets. Implantation of the microencapsulated islet graft into the peritoneal cavity was carried out under ether anaesthesia, with a 16 gauge cannula attached to a $10 \mathrm{ml}$ syringe. The abdomen was closed with a two-layer suture. Rats which received non-encapsulated allografts were implanted with 2800-3000 islets, with a total graft volume of $8.0-10.0 \mu 1$. These islets were injected into the peritoneal cavity through a 23 gauge butterfly needle. Non- fasting blood glucose and body weight were frequently determined at regular intervals, as indicated in Figures 1 and 2. Blood was taken from the tail vein under ether anaesthesia and the glucose concentration was determined with glucose test tapes (Reflolux, Boerhinger, Mannheim, FRG). Daily urine output was measured in perspex metabolic cages 1 week before, and 2-4 weeks after transplantation. Urine glucose excretion was estimated with urine glucose test tapes (Glukotest, Boerhinger).

At 4 weeks after implantation, a biopsy of the pancreas of recipient animals was taken (approximately $50 \mathrm{mg}$ of the tail) to determine whether Beta-cell regeneration had occurred. Aldehyde-fuchsin positive Beta-cells in the native islets were counted and expressed as percentage of the total number of cells per islet. Before streptozotocin treatment, $65 \%$ Beta-cells were present in the islets. Per biopsy, at least 10 islets in four different sections were evaluated.

\section{Cannulation surgery and glucose tolerance tests}

At 5-6 weeks after transplantation, glucose tolerance was tested by determining glucose and insulin levels during intravenous glucose infusion (IVGTT) and after test meal administration. Only normoglycaemic animals, with regained body weight and less than $3 \%$ aldehyde-fuchsin positive Beta-cells in their native pancreas, were provided with two permanent intracardiac catheters, implanted via the right and left jugular vein into the right atrium $(n=4)$. These two catheters allowed blood sampling as well as intracardiac glucose infusion in unstressed, freely moving rats [15]. Normal healthy rats served as controls $(n=5)$, with an average body weight similar to that of the animals with encapsulated islet grafts. IVGTTs were performed by intracardiac infusion of $200 \mathrm{mg}$ glucose at a rate of $10 \mathrm{mg} / \mathrm{min}$. The meal tests were carried out by offering the animals $2.0 \mathrm{~g}$ pelleted rat chow, after the animals had been habituated to ingesting the offered meal within 5 min [16]. To establish baseline values, blood samples were taken at $10 \mathrm{~min}$ and immediately before the start of the test, and at regular intervals thereafter, as indicated in the figures. Each time of blood sampling, $0.3 \mathrm{ml}$ blood for blood glucose and plasma insulin determination was taken from one of the two indwelling heart catheters. After every third blood sample, $0.9 \mathrm{ml}$ blood from a syngenic donor rat was injected into the same catheter, in order to avoid stress due to blood loss. Blood glucose concentrations were determined in whole blood by a ferricyanide method with a Technicon autoanalyzer. Plasma insulin was measured by radio-immuno-assay, with rat insulin used as a standard, as we previously described [17]. Antibody-bound insulin precipitation was carried out with poly-ethylene-glycol [18].

\section{Histological evaluation of microencapsulated islet grafts}

At 5 weeks after transplantation, microcapsules were retrieved by peritoneal lavage from one animal which died after cannulation surgery. At the same time, a biopsy was taken from the omentum to examine whether there were adherent microcapsules. Microcapsules were also retrieved by peritoneal lavage and omental biopsies from the five animals which had again become hyperglycaemic at about 1-2 weeks after the recurrence of hyperglycaemia, and from the three persistingly normoglycaemic animals at the time when they were killed ( 4 months after transplantation). Simultaneously, a second pancreas biopsy was taken from these animals to confirm the absence or presence of native Beta-cell regeneration. Recovered capsule specimens were fixed in Bouin's solution for paraffin processing, and stained with aldehyde fuchsin.

\section{Statistical analysis}

The Student's $t$-test was applied to determine significant differences in daily urine excretion, blood glucose and insulin levels. Values are given as mean \pm SEM. Differences were considered to be statistically significant if $p<0.05$. 


\section{Results}

Intraperitoneal implantation of non-encapsulated islet allografts in diabetic rats $(n=5)$ did not reverse hyperglycaemia (Fig. 1). In these rats, only a temporary decrease in blood glucose was observed, and all were again severely hyperglycaemic at 1 week after transplantation (blood glucose $>20 \mathrm{mmol} / \mathrm{l}$ ).

In contrast, non-fasting blood glucose levels in animals transplanted with microencapsulated allografts normalized in all cases (Fig.1). The average time after which the animals became normoglycaemic was $7 \pm 1$ days. At 4 weeks after implantation, pancreas biopsies from native pancreases showed Beta-cell regeneration in three animals $(13 \pm 4 \%$ aldehyde fuchsin positive Beta-cells in the islets). These animals remained normoglycaemic for more than 4 months. One animal which died after cannulation surgery, had low blood glucose levels $(4.0 \mathrm{mmol} / 1)$, and another animal became hyperglycaemic at 5 weeks after transplantation. The remaining four animals continued to be normoglycaemic for 10-14 weeks, and were subjected to intracardial cannulation and glucose tolerance testing.

The normalization of body weight of animals with microencapsulated islet grafts corresponded well with the normalization of the blood glucose levels (Fig.2). After streptozotocin treatment, all diabetic animals lost about $20 \%$ of their body weight. Rats with non-encapsulated allografts showed only a short period of weight gain, whereas diabetic controls totally failed to gain weight. In contrast, rats with microencapsulated allografts immediately showed body weight gain after implantation of the islets, which was maintained during their normoglycaemic period. This metabolic improvement was also demonstrated by a dramatic fall in urinary output from $95.5 \pm$ $14.7 \mathrm{ml} / 24 \mathrm{~h}$ before to $8.2 \pm 1.6 \mathrm{ml} / 24 \mathrm{~h}$ after transplantation. However, urinary glucose excretion was not com-

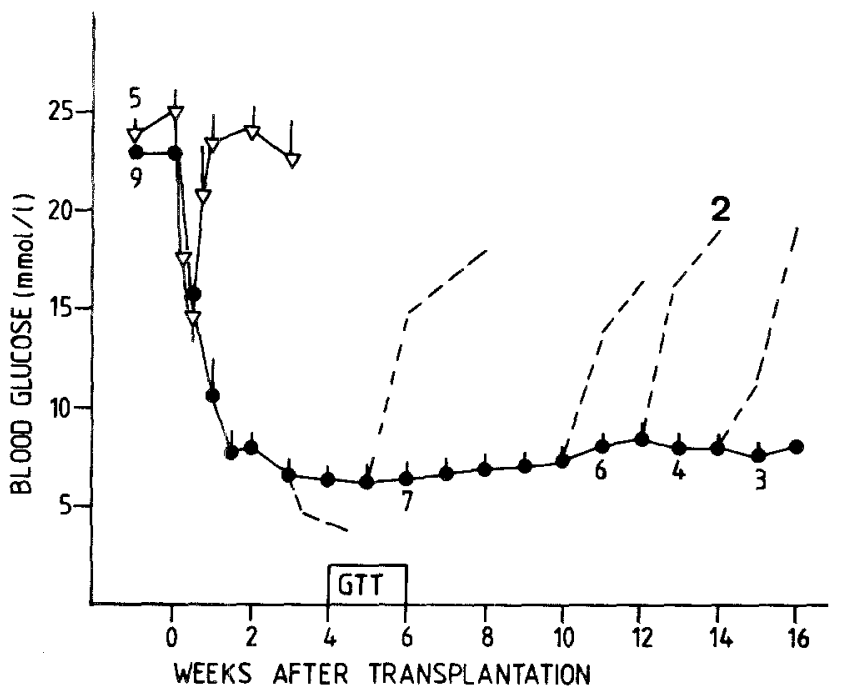

Fig. 1. Non-fasting blood glucose concentrations in streptozotocin diabetic rats after intraperitoneal implantation of microencapsulated islet allografts $(\bullet, n=9)$, and non-encapsulated islet allografts ( $\nabla-\nabla, n=5$ ). The number of animals (as indicated) decreased due to death, and to recurrence of hyperglycaemia (see Results). $\mathrm{GTT}=$ glucose tolerance tests

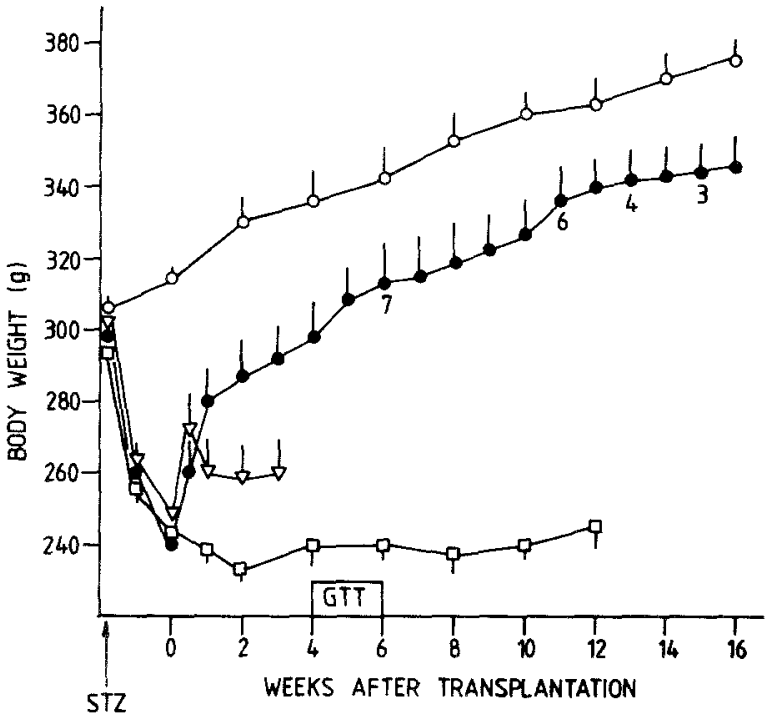

Fig. 2. Changes in body weight in diabetic rats after intraperitoneal implantation of microencapsulated islet allografts $(\bullet-\bullet, n=9)$, and non-encapsulated islet allografts ( $\vee-\nabla, n=5)$; diabetic control animals ( $\square-\square, n=6)$; normal healthy control animals $(O-O, n=6)$. The number of animals (as indicated) decreased due to death, and to recurrence of hyperglycaemia (see Results). STZ = streptozotocin, $\mathrm{GTT}=$ glucose tolerance test

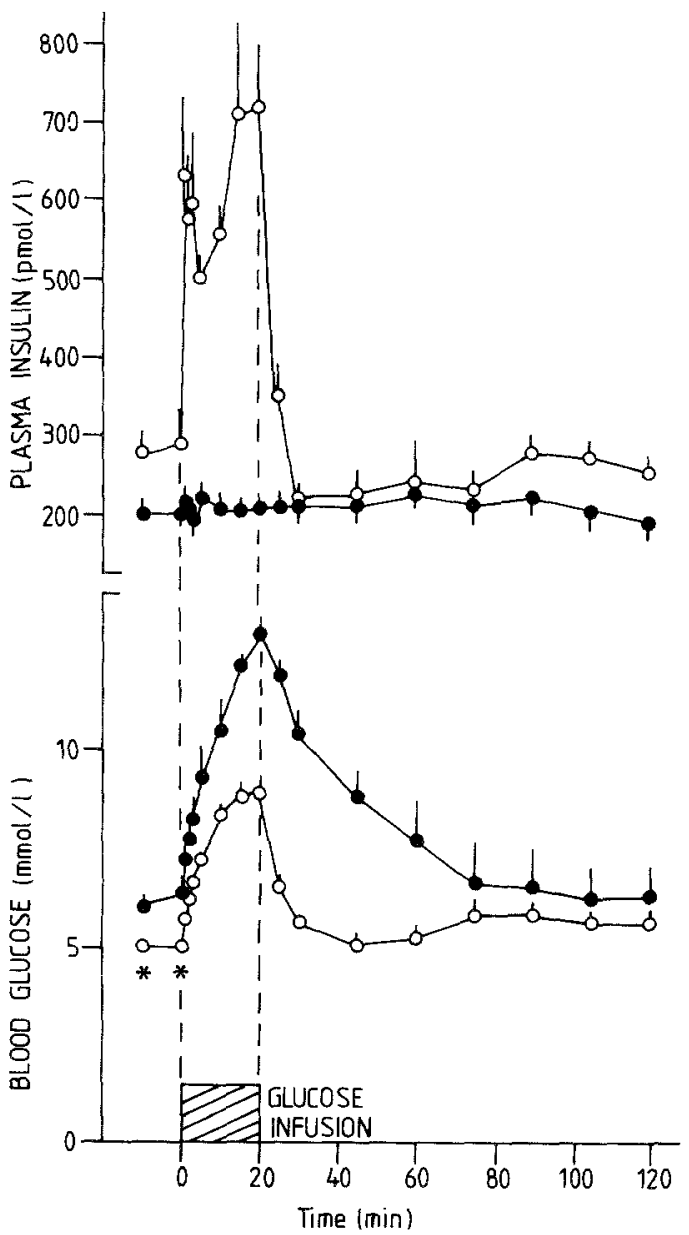

Fig.3. Blood glucose and plasma insulin levels after intravenous glucose infusion $(10 \mathrm{mg} / \mathrm{min})$ in rats with intraperitoneally implanted microencapsulated islet allografts $(\bullet, n=4)$, and normal healthy controls $(0-0, n=5) \cdot{ }^{*} p<0.05$ 


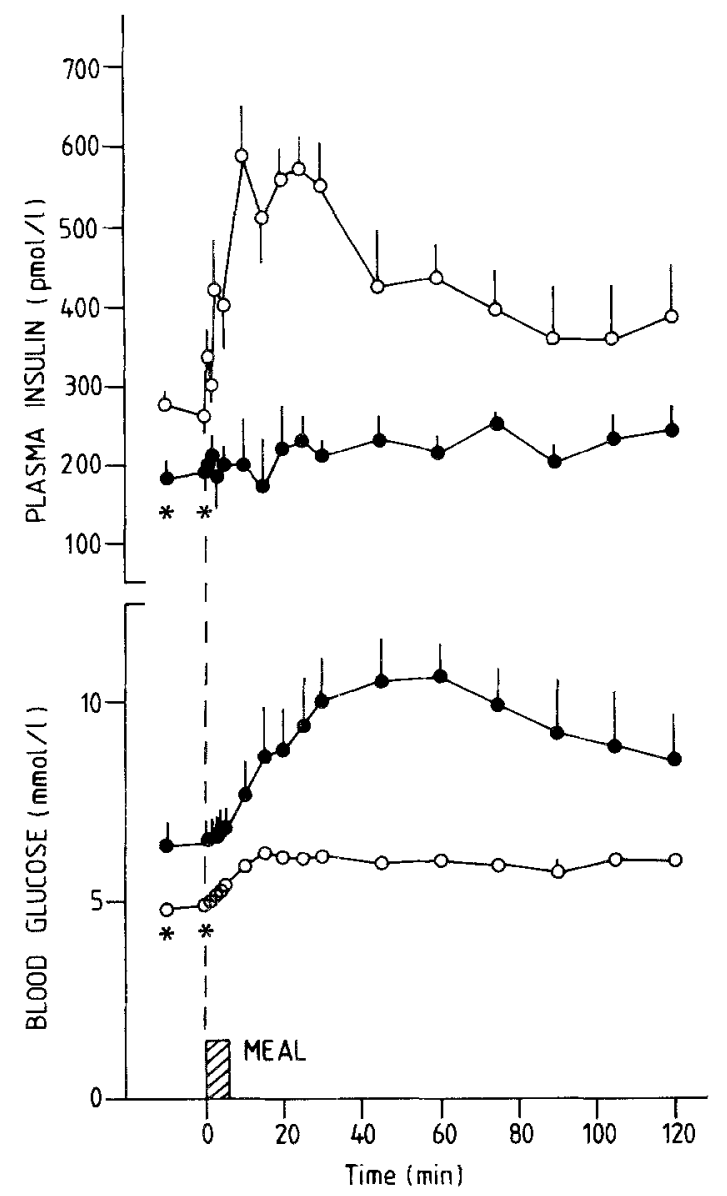

Fig. 4. Blood glucose and plasma insulin levels after spontaneous ingestion of a test meal in rats with intraperitoneally implanted microencapsulated islet allografts $(\bullet, n=4)$, and in normal healthy control animals $(0-0, n=5) .{ }^{*} p<0.05$

pletely eliminated, since it was $\geq 28 \mathrm{mmol} / 1$ before and $0-2.2 \mathrm{mmol} / \mathrm{l}$ after transplantation.

Prior to the glucose tolerance tests, basal glucose levels in rats with microencapsulated islets were somewhat higher than in normal control animals $(p<0.05)$, which corresponded with the concomitant basal plasma insulin levels, since these were found to be lower in rats with microencapsulated islets than in normal controls (Figs. 3 and 4). Maximum blood glucose levels were significantly higher in rats with microencapsulated islets than in normal control rats, both during glucose infusion (13.0 \pm 0.4 and $8.9 \pm 0.4 \mathrm{mmol} / \mathrm{l}$, respectively, $p<0.01)$ and after test meal administration $(10.6 \pm 0.9$ and $6.2 \pm 0.1 \mathrm{mmol} / \mathrm{l}$, respectively, $p<0.001$ ). This is in accordance with the concomitant plasma insulin, which showed hardly any increase in rats with microcapsules, whereas normal control animals showed a considerable plasma insulin elevation with both test situations.

On histological examination, microcapsules retrieved by peritoneal lavage from the animal which died after cannulation surgery ( 5 weeks after transplantation) were found to contain viable islets with aldehyde-fuchsin positive Beta-cells (Fig. 5 a). These microcapsules were intact and largely free from cellular overgrowth. However, when the omentum of this animal was histologically examined, other microcapsules were found to be adherent to the omentum and overgrown with fibrous tissue (Fig. 5 b). These capsules, which could not be flushed out by peritoneal lavage, contained non-viable islet remnants.

Histological examination of microcapsules retrieved from animals which had again become hyperglycaemic showed many capsules overgrown with fibrotic tissue and few capsules free from cellular overgrowth. In these animals, neither capsules with fibrotic overgrowth, nor capsules free from cellular overgrowth contained viable islet tissue. In contrast, capsules retrieved from animals still normoglycaemic at the time of peritoneal lavage, contained viable aldehyde-fuchsin positive Beta-cells, though only when the capsule surface was free from cellular overgrowth.

The histological appearance of the pancreases at the end of the study confirmed the observations of the previously taken pancreas biospies. Hyperglycaemic recipients showed no aldehyde fuchsin positive Beta-cells, whereas the animals with persistent normoglycaemia showed considerable Beta cell regeneration, although to a lesser extent than in their first pancreas biopsy $(8.5 \pm 2 \%$ aldehyde fuchsin positive Beta-cells in the islets).
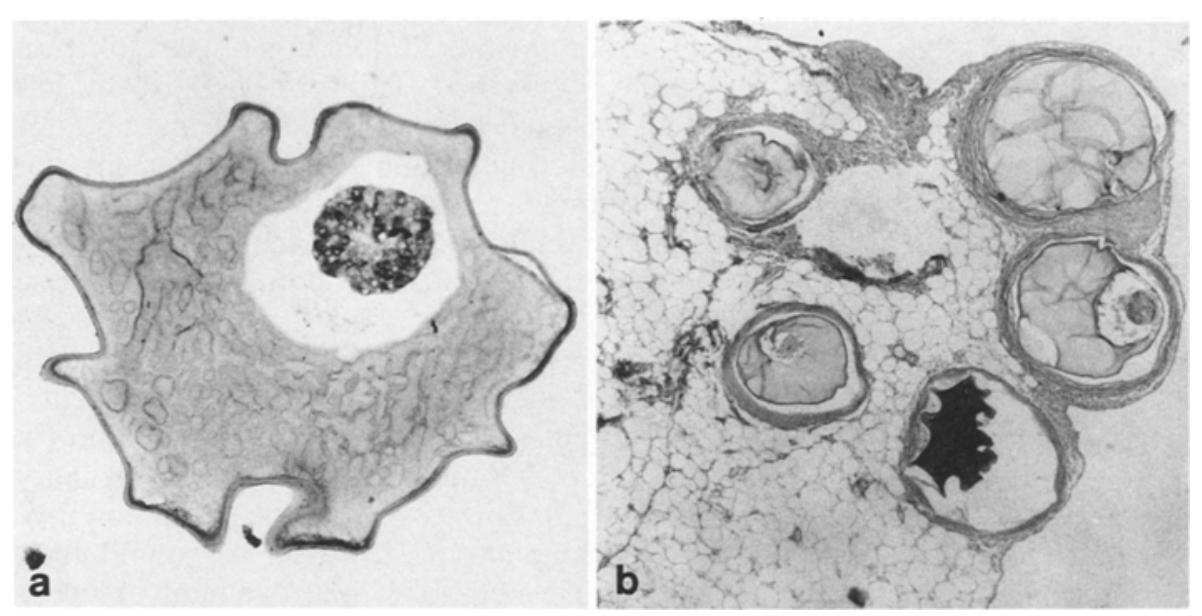

Fig. 5a,b. Alginate polylysine microencapsulated rat islets recovered from the peritoneal cavity at 5 weeks after allo-transplantation. a Aldehyde fuchsin, $100 \times, \mathbf{b}$ Microcapsules in omental fat tissue, aldehyde fuchsin, $200 \times$ 


\section{Discussion}

The present study shows that normoglycaemia can be achieved after transplantation of microencapsulated rat is let allografts into streptozotocin diabetic recipients, in the absence of immunosuppressive therapy. These results thereby confirm other studies on islet microencapsulation $[6-8,10]$. We found that implantation of non-encapsulated islets led to only a temporary decrease of blood glucose levels, followed by a quick return to hyperglycaemia. It is likely that these islets were subject to allograft rejection, which demonstrates the immunoprotective properties of the alginate-polylysine membrane in case of the encapsulated islet graft. These immunoprotective properties were further substantiated by the retrieval of histologically viable and intact islets within microcapsules from normoglycaemic recipients. However, our study also extends previous observations by demonstrating that, in spite of persisting normoglycaemia, the kinetic responses to oral and intravenous glucose challenge were far from normal.

Spontaneous Beta-cell regeneration in the host native pancreas may occur after induction of diabetes with streptozotocin [19-21]. Indeed, at 1 month after transplantation we found a considerable number of aldehyde-fuchsin positive Beta-cells in the native pancreases in three out of nine recipients. A similar observation was made by Chicheportiche et al. [22], who found extensive regeneration in the recipient pancreas after implantation of microencapsulated islet xenografts. Since we could not rule out a contribution of regenerated native Beta cells to the prolonged normoglycaemia, these three animals were not subjected to further evluation by glucose tolerance testing. The normoglycaemia observed in the other animals could not be attributed to native Beta-cell regeneration, since this was histologically excluded.

These animals showed that the microencapsulated islet allograft was quite effective in restoring normoglycaemia and sufficiently adequate to normalize both weight gain and body weight as well as the volume of urine output. This is in contrast to a previous study in which it was demonstrated that the increase of body weight is only limited to the first few weeks following transplantation [10]. The low insulin increments during glucose tolerance testing seem to indicate that the observed non-fasting normoglycaemia was achieved by only basal release of insulin from the encapsulated isiet graft. A similar effect on blood glucose in streptozotocin diabetic rats can be obtained by the implantation of insulin containing polymer matrices [2325]; devices which release insulin at a basal rate without any response to blood glucose elevation. However, there is a principal difference in kinetic performance since we demonstrated in a previous study that glycaemic stimulation of microencapsulated islets results in a rapid and significant insulin response when tested in vitro [14]. Thus, other factors should be considered to explain for the lack of insulin response by microencapsulated islets after transplantation.

One factor is that unfavourable glucose and insulin diffusion kinetics over the membrane may have contributed to the reduced insulin responses, since we used slightly larger microcapsules in this study than in our previous in vitro experiments [26]. This effect may have been further enhanced by the fact that the majority of rat islets, isolated from one donor pancreas, have small diameters [27] and consequently relativily large capsules.

A second factor to consider is the total volume of the grafted islet tissue in relation to the transplantation site. In a recent study, we demonstrated that intraperitoneal implantation of free isogenic islets with a total islet tissue volume of $8.0-10.0 \mu \mathrm{l}$ normalizes blood glucose levels in diabetic rats routinely, and restores adequate glucose metabolism and insulin responses during IVGTT and test meal [28]. However, the principal difference between free and microencapsulated islet grafts is that free islets become attached to the abdominal viscera and become completely vascularized, while microcapsules freely float across the peritoneal cavity, without any vascular access. This implies that a delay of glucose and insulin exchange between the peritoneal fluid and the blood may have consequences for the magnitude and the rapidity of the plasma insulin response. For instance, it has been demonstrated that the intraperitoneal glucose concentration in rats follows the blood glucose with a 5 min delay [29]. On the other hand, also the rate of absorption of intraperitoneally delivered insulin into the circulation was found to be time-dependent [30], and directly related to the secreted amount of insulin [31]. Whether the amount of insulin, maximally secreted by $8.0-11.0 \mu$ lencapsulated islet tissue, is sufficient to induce an adequate plasma insulin response is questionable $[14,32,33]$, and has to be further investigated.

A final factor relating to the impaired glucose tolerance may be a gradual decrease of the number of viable and functioning islets after transplantation. Some observations substantiate this hypothesis. First, at 5 weeks after implantation many islet-containing capsules could be retrieved with peritoneal lavage, but it was also found that some capsules were attached to the omentum, surrounded by a fibrotic reaction and containing non-viable islet remnants. Second, all animals that had been normoglycaemic in the absence of native Beta-cell regeneration successively returned to hyperglycaemia in the course of 5 to 14 weeks. From these animals no viable islets could be retrieved. Finally, peritoneal lavage in animals which had maintained prolonged normoglycaemia in the presence of native Beta-cell regeneration yielded only few islet-containing capsules free from cellular overgrowth.

The main reason for the gradual loss of islet function seems to be the occurrence of fibrotic overgrowth, impeding the diffusion of hormones and metabolites through the capsule membrane. An additional mechanism may well be that, as a consequence of gradual loss of islet mass, the remaining islets within the non-overgrown capsules are subject to exhaustion and ultimate decrease of viability.

In conclusion, our data show that implantation of microencapsulated islets effectively restores normoglycaemia, urine output, and weight gain in streptozotocin diabetic rats for up to 4 months, and that the alginatepolylysine membrane can provide adequate immunoisolation after transplantation. However, we found that, despite the major metabolic improvement, glucose 
tolerance remained severely impaired, because the encapsulated islet graft was not able to increase plasma insulin levels adequately in response to blood glucose elevation. Relevant factors in this regard are the size of the microcapsules applied, the total volume of the encapsulated islet tissue, and a gradual decrease in graft viability.

Acknowledgements. The authors are greatfully indebted to Mrs. M. A. Vonk for performing the radio-immuno-assays, and to Mr. A. Pasma and Dr. P.O. Gerrits for the histological preparations. Financial support for this study was obtained from the Diabetes Research Fund and the Jan Kornelis de Cock Foundation, The Netherlands.

\section{References}

1. Gray DWR, Morris PJ (1986) Prospects for pancreatic islet transplantation. World J Surg 10: 410-421

2. Gray DWR, Morris PJ (1987) Developments in isolated pancreatic islet transplantation. Transplantation 43: 321-331

3. Hering BJ, Bretzel RG, Federlin K (1988) Current status of clinical islet transplantation. Horm Metab Res 20:537-545

4. Reach G (1990) Bioartificial pancreas: Status and bottlenecks. Int J Artif Organs 13: 329-336

5. Darquy S, Reach G (1985) Immunoisolation of pancreatic B cells by microencapsulation. Diabetologia 28: 776-780

6. Lim F, Sun AM (1980) Microencapsulated islets as bioartificial endocrine pancreas. Science 210: 908-910

7. O'Shea GM, Goosen MFA, Sun AM (1984) Prolonged survival of transplanted islets of Langerhans encapsulated in a biocompatible membrane. Biochim Biophys Acta 804: 133-136

8. Sun AM, O'Shea GM (1985) Microencapsulation of living cells, a long-term delivery system. J Controlled Release 2: 137-141

9. O'Shea GM, Sun AM (1986) Encapsulation of rat islets of Langerhans prolongs xenograft survival in diabetic mice. Diabetes 35: 943-946

10. Fan MY, Lum ZP, Fu XW, Levesque L, Tai IT, Sun AM (1990) Reversal of diabetes in BB rats by transplantation of encapsulated pancreatic islets. Diabetes 22: 519-522

11. Weber CJ, Zabinski S, Koschitzky T, Wicker L, Rajotte R, D'A gati V, Peterson L, Norton J, Reemtsma K (1990) The role of CD4 + helper T-cells in the destruction of microencapsulated islet xenografts in NOD mice. Transplantation 49:396-404

12. Van Suylichem PTR, Wolters GHJ, Van Schilfgaarde R (1990) The efficacy of density gradients for islet purification: a comparison of seven density gradients. Transplant Int 3: 156-161

13. Lim F (1984) Microencapsulation of living cells and tissues, theory and practice. In: F.Lim (ed) Biomedical applications of Microencapsulation. Boca Raton, FL, CRC-Press, pp 137-154

14. Fritschy WM, Wolters GHJ, Van Schilfgaarde R (1991) Effect of alginate polylysine alginate microencapsulation on in vitro insulin release from rat pancreatic islets. Diabetes 40:37-43

15. Steffens AB (1969) A method for frequent sampling of blood and continuous infusion of fluids in the rat without disturbing the animal. Physiol Behaviour 4: 833-836

16. Strubbe JH, Steffens AB (1975) Rapid insulin release after ingestion of a meal in the unanesthetized rat. Am J Physiol 229: 10191022

17. Bosboom RS, Zweens J, Bouman PR (1973) Effects of feeding and fasting on the insulin secretory response to glucose and sulfonylureas in intact rats and isolated perfused rat pancreas. Diabetologia 9:243-250
18. Desbuquois B, Aurbach GD (1971) Use of polyethylene glycol to separate free and antibody-bound peptide hormones in radioimmuno assays. J Clin Endocrinol Metab 33: 732-738

19. Trimble ER, Karakash C, Malaisse-Lagae F, Vassutine I, Orci L, Renold AE (1980) Effects of intraportal islet transplantation on the transplanted tissue and the recipient pancreas. Functional studies. Diabetes 29: 341-347

20. Kruszynska YT, Home PD, Morley A, Alberti KGMM (1986) Preservation of islet function and morphology after transplantation into high dose streptozotocin diabetic rats. Diab Res 3: 175181

21. Hahn HJ, Lucke S, Ziegler B, Besch W, Kauert C(1990) Effect of islet transplantation on the recipient endocrine pancreas. Biomed Biochim Acta 44: 137-142

22. Chicheportiche D, Darquy S, Lepeintre J, Capron F, Halban PA, Reach $G$ (1990) High-performance liquid chromatography analysis of circulating insulins distinguishes between endogenous insulin production (a potential pitfall with streptozotocin diabetic rats) and islet xenograft function. Diabetologia 33: 457-461

23. Creque HM, Langer R, Folkman J (1980) One month of sustained release of insulin from a polymer implant. Diabetes 29 : $37-40$

24. Brown L, Munoz C, Siemer L, Edelman E, Langer R (1986) Controlled release of insulin from polymer matrices. Control of diabetes in rats. Diabetes 35: 692-697

25. Wang PY (1987) Prolonged release of insulin by cholesterol matrix implant. Diabetes 36: 1068-1072

26. Chicheportiche D, Reach $G$ (1988) In vitro kinetics of insulin release by microencapsulated rat islets: effect of the size of the microcapsules. Diabetologia 31: 54-57

27. Wolters GHJ, Van Suylichem PTR, Pasma A, Van Schilfgaarde R (1990) Morphometric analysis of the endocrine pancreas of rats and normal and hyperglycemic mice. Neth $\mathrm{J}$ Med 36: A1 (Abstract)

28. Fritschy WM, Van Straaten JFM, De Vos P, Strubbe JH, Wolters GHJ, Van Schilfgaarde R (1991) The efficacy of intraperitoneal pancreatic islet isografts in the reversal of diabetes in rats. Transplantation (in press)

29. Velho G, Froguel Ph, Reach G (1989) Determination of peritoneal glucose kinetics in rats: implications for the peritoneal implantation of closed-loop insulin delivery systems. Diabetologia 32: $331-336$

30. Schade DS, Eaton RP, Davis T, Akiya F, Phinney E, Kubica R, Vaugn EA, Day PW (1981) The kinetics of peritoneal insulin absorption. Metabolism 30: 149-155

31. Rubin J, Bell AH, Andrews M, Jones Q, Planch A (1989) Intraperitoneal insulin - a dose response curve. Trans Am Soc Artif Intern Organs 35: 17-21

32. Wolters GHJ, Konijnendijk W, Bouman PR (1977) Effects of fasting on insulin secretion, islet glucose metabolism, and the cyclic adenosine $3^{\prime}, 5^{\prime}$-monophosphate content of rat pancreatic islets in vitro. Diabetes 26:530-537

33. Poulsen JSD, Smith M, Deckert M, Deckert T (1980) Comparison of intraperitoneal, intraportal and intravenous insulin infusion. Acta Endocrinol 95: 500-504

Received: 18 February 1991

and in revised form: 18 April 1991

Dr. W.M.Fritschy

Surgical Research Laboratory

Department of Surgery

University of Groningen

Bloemsingel 1

NL-9713 BZ Groningen

The Netherlands 